jofssm_0501_richards\&smith.doc?CFID=893038\&CFTOKEN= 10071954 (accessed 3 August 2008).

Rimli, Lisa. 2008. "Case Study Angola." Pp. 38-60 in Ulrike Joras and Adrian Schuster (eds.) Private Security Companies and Local Populations: An Exploratory Study of Afghanistan and Angola. Working Paper 1/2008.
Schmeidl, Susanne. 2007. "Case Study Afghanistan - Who Guards the Guardians?" Pp.14-45 in Private Security Companies and Local Populations: An Exploratory Study of Afghanistan and Angola. Swisspeace report, November 2007.

. 2008. "”Case Study Afghanistan." Pp.9-38 in Ulrike Joras and Adrian Schuster (eds.) Private Security Companies and Local Populations: An Exploratory Study of Afghanistan and Angola. Working Paper 1/2008.

\title{
Die Selbstregulierung privater Sicherheits- und Militärfir- men als Instrument der Marktbeeinflussung
}

\author{
Andrea Schneiker*
}

\begin{abstract}
Since the beginning of the 1990s, the number of private security and military firms (PSMFs) has grown rapidly. PSMFs are profit-driven companies that sell military, police and intelligence services. Today, PSMFs are charged with the provision of security in war and post-war situations, although PSMF operations may also contribute to greater levels of violence. Hence the need for PSMFs to be more strictly regulated. Most observers discuss existing national legislation and assess its shortcomings. This article analyses the self-regulation of PSMFs by discussing how PSMFs seek to influence the market for their services and the behaviour of their clients.
\end{abstract}

Keywords: Security, privatization, self-regulation, conduct codes

\section{Einleitung ${ }^{1}$}

S pätestens seit dem jüngsten Skandal um die Firma Blackwater im Irak ist die Debatte über die Regulierung privater Sicherheits- und Militärfirmen (PSMFs) ${ }^{2}$ neu entbrannt. Zur Erinnerung: Am 16. September 2007 hatten Angestellte von Blackwater während einer Schießerei in Bagdad mindestens 14 Zivilisten grundlos erschossen (vgl. Johnston/Broder 2007). Daraufhin wollte die irakische Regierung Blackwater des Landes verweisen, musste sich aber dem Willen der US-Regierung fügen, die im Irak auf die Sicherheitsdienstleistungen von Blackwater angewiesen ist (vgl. Broder/Risen 2007). Doch nicht nur im Irak, sondern global haben sich PSMFs zu einem bedeutenden Akteur in Kriegs- und Nachkriegssituationen entwickelt.

Unter PSMFs werden hier international operierende Firmen verstanden, die verschiedene militärische, polizeiliche und/ oder nachrichtendienstliche Tätigkeiten durchführen. Diese lassen sich in neun Kategorien unterteilen: Beratung, Training und Ausbildung, Logistik, technische Dienste, Minenräumung und Waffenentsorgung, Demobilisierung und Reintegration ehemaliger Kämpfer, Aufklärung, bewaffneter Personen-, Ge-

Referierter Beitrag.

* Dipl.-Soz.Wiss. Andrea Schneiker ist wissenschaftliche Mitarbeiterin am Institut für Politische Wissenschaft der Leibniz Universität Hannover.

1 Für viele konstruktive Anmerkungen danke ich Željko Branovic, André Bank, Jens Taken und den beiden Gutachtern.

2 Andere gängige Bezeichnungen sind: private security companies (PSCs) bzw. private Sicherheitsfirmen, private military companies (PMCs) bzw. private Militärfirmen. bäude- und Konvoischutz und Kampfeinsätze. ${ }^{3}$ Kunden von PSMFs sind hauptsächlich Regierungen, allen voran die der USA, sowie internationale Regierungsorganisationen (IGOs), Nichtregierungsorganisationen (NGOs) und Unternehmen.

Derzeit gibt es über 300 solcher PSMFs ${ }^{4}$ (vgl. Messner/Gracielli 2007: 13), von denen die meisten in den USA und in Großbritannien beheimatet sind. Die hauptsächlichen Einsatzgebiete der Firmen sind Kriegs- und Nachkriegsgebiete. In diesen führen PSMFs vermehrt Dienstleistungen im Rahmen von (Wieder-)Aufbau- und Statebuildingprogrammen durch. Kern ihrer Arbeit ist dabei die Bereitstellung von Sicherheit im Einsatzgebiet. Sicherheit wird hier verstanden als die Abwesenheit von oder der Schutz vor existenziellen Bedrohungen, die physische Gewalt beinhalten. Dabei lassen sich zwei zentrale Tätigkeitsbereiche identifizieren, die auch in Zukunft eine wichtige Rolle spielen werden (vgl. Bearpark/Schulz 2007: 240f.; Donald 2006: 45ff.): erstens der bewaffnete Personen-, Gebäude- und Konvoischutz für nationale und ausländische Regierungen, Streitkräf-

3 In der Literatur finden sich verschiedene Ansätze, PSMFs zu typologisieren. Am häufigsten rezipiert wird die „Speerspitzen-Typologie“ Peter Singers (2006: 156ff.). Danach werden PSMFs entsprechend ihrer Nähe zum Kampfgeschehen bzw. ihrer Position auf dem Kriegsschauplatz in drei Kategorien eingeteilt: Militärdienstleister (Military Provider Firms), militärische Beratungsfirmen (Military Consultant Firms) und militärnahe Dienstleister (Military Support Firms). Eine Zuordnung der Firmen zu diesen drei Kategorien ist jedoch angesichts der Tatsache, dass viele PSMFs sehr unterschiedliche Dienstleistungen anbieten und dabei in mehreren der drei Bereiche aktiv werden, schwierig. Daher wird für die hier diskutierte Frage auf diese Typologie verzichtet.

4 Auf Grund der Intransparenz der Branche (nicht alle Firmen sind offiziell registriert) und der Mobilität der Firmen (Fusionen, Verlegung des Firmensitzes, Namensänderung etc.) kann deren Anzahl nur geschätzt werden. 
te, IGOs, NGOs und Unternehmen, zweitens die Beratung und Ausbildung staatlicher Sicherheitsakteure aus den Bereichen Polizei, Militär und Justiz, die teilweise im Rahmen von Programmen zur Sicherheitssektorreform (SSR) stattfindet.

Diese eigentlich einem Nachkriegskontext zugeordneten Aufgaben finden jedoch zunehmend in (Noch-)Kriegssituationen statt, zum Beispiel in Afghanistan und im Irak. Dabei tragen PSMFs jedoch nicht nur zu Sicherheit, sondern auch zu Unsicherheit oder gar zu Gewalt bei. Entsprechend dem hiesigen Sicherheitsverständnis existiert Unsicherheit, solange es keinen effektiven Schutz vor einer existenziellen Bedrohung gibt oder diese nicht abgewendet wurde. Ein Grund für den Beitrag von PSMFs zu Unsicherheit und Gewalt ist deren zunehmende Bewaffnung, die aus der Nähe zum Kampfgeschehen herrührt. Dabei wird vor allem einigen im Irak operierenden PSMFs vorgeworfen, systematisch Gewalt zu präventiven Zwecken einzusetzen und dabei Zivilisten zu töten, beispielsweise durch das wahllose Abfeuern von Maschinengewehrsalven (vgl. Finer 2005; Miller 2005; Singer 2007: 6). Derartige Vorgehensweisen schüren Angst und Ablehnung bei der lokalen Bevölkerung und erzeugen Gegengewalt. Da die Bevölkerung meist nicht zwischen ausländischen PSMFs und regulären ausländischen Streitkräften unterscheiden kann, richtet sich diese Gewalt auch gegen Letztere. Darüber hinaus verwandeln PSMFs auch zivile Akteure in Angriffsziele, indem sie die Grenzen zwischen militärischen Peace-Enforcement-Operationen, humanitärer Hilfe und Maßnahmen zum Wiederaufbau in den Augen weiter Teile der Bevölkerung verwischen. Wenn z.B. die Angestellten eines Unternehmens, das mit dem Ausbau einer Straße betraut ist, von bewaffneten PSMF-Angestellten bewacht werden, dann ist diese zivile Wiederaufbaumaßnahme nicht unbedingt als solche erkennbar. Noch dazu, wenn PSMFs (es müssen nicht dieselben sein) in anderen Situationen militärische Konvois begleiten oder regulären Streitkräften bei Übergriffen Schützenhilfe leisten (vgl. Bjork/Jones 2005: 784ff.). Somit werden zivile Akteure durch die Anwesenheit von PSMFs zu Angriffszielen, obwohl die Anwesenheit der PSMFs eigentlich ihrem Schutz dienen sollte. Dadurch wiederum rechtfertigen PSMFs ihre bisherigen und weiteren Einsätze. Die Unsicherheit und der sich daraus ergebende Bedarf an PSMFs werden somit zur „self-fulfilling prophecy“, die im Irak von einer anziehenden Gewaltspirale begleitet wurde (vgl. Bjork/Jones 2005: 789ff.).

Um derart negative Auswirkungen der Einsätze von PSMFs zu verhindern oder zumindest die PSMF-Angestellten für ihr Handeln zur Verantwortung zu ziehen, wird oft gefordert, die Einsätze von PSMFs einer (strikteren) Regulierung zu unterziehen. Dabei herrscht weitgehend Einigkeit darüber, dass es keine „One-size-fits-all-Lösung“ geben kann, sondern Regulierung auf mehreren Ebenen unter Beteiligung verschiedener Akteure stattfinden muss. Die Hauptverantwortung für eine Regulierung wird dabei bei den Staaten gesehen und staatliche Regulierung somit als zentral erachtet. Da die Einsatzländer (host states) der PSMFs selbst im Falle einer existierenden gesetzlichen Grundlage meist über keinen (effektiven) Justizapparat verfügen, bleibt die Regulierung in der Praxis meist den Auftrag gebenden Staaten (client states) oder den Heimatstaaten der PSMFs (home states) überlassen. Von diesen verfügen jedoch nur sehr wenige (v.a. USA, Südafrika, Israel) über PSMF-spezifische
Regulierungsinstrumente. In der Literatur wurde die Umsetzung dieser Gesetze ausführlich analysiert, ihre Schwächen wurden beschrieben und Verbesserungsvorschläge unterbreitet (vgl. z.B. Avant 2005; Caparini 2007; Schreier/Caparini 2005; Singer 2004). Im Rahmen der sogenannten Schweizer Initiative diskutieren seit Anfang 2006 Staatenvertreter über staatliche Regulierung von PSMFs ${ }^{5}$, ohne dass bisher eine solche beschlossen worden wäre.

Eine freiwillige Selbstregulierung von PSMFs kann Staaten nicht aus ihrer Verantwortung entlassen und nationale oder internationale Regulierung nicht ersetzen, sondern sollte als komplementäres Regulierungsinstrument verstanden werden. Diskutiert werden müsste, nicht zuletzt auf politischer Ebene, welche Aspekte Selbstregulierung verregeln soll und wie eine solche Regulierung in ein mehrere Ebenen umfassendes Regulierungssystem eingebunden werden könnte. Hier wird jedoch untersucht, welche Möglichkeiten die Selbstregulierung den PSMFs zur Einflußnahme bietet. Dabei liegt zunächst die Frage nach der Einflussnahme auf politische Akteure, Prozesse und Inhalte auf der Hand. Es ist zu vermuten, dass PSMFs bestrebt sind, zukünftige staatliche Regulierung derart zu beeinflussen, dass diese ihre Arbeit nicht behindert. Doch da es noch keine nationale Regulierung gibt, die mittels Selbstregulierung entsprechend beeinflusst wurde, lässt sich auch keine solche Einflussnahme nachweisen. Es kann aber untersucht werden, inwiefern Selbstregulierung Möglichkeiten der Einflussnahme auf den Markt für PSMF-Dienstleistungen und deren Kunden bietet.

Dabei geht es PSMFs einerseits darum, Selbstregulierung als Kennzeichen für respektable Firmen („upscale firms“) und somit eine Abgrenzung zu schwarzen Schafen („downscale firms“) ${ }^{6}$ zu etablieren (vgl. Schulz 2008: 161). Darauf aufbauend soll andererseits die Auftragsvergabe durch die Kunden derart beeinflusst werden, dass jene nur Firmen unter Vertrag nehmen, die sich einer Selbstregulierung unterziehen. Dazu wird im Folgenden zunächst die Differenzierung zwischen „upscale firms“ und „downscale firms“ dargestellt. Anschließend wird die doppelte Strategie der Einflussnahme von PSMFs mittels Selbstregulierung im Allgemeinen diskutiert, wobei im Speziellen Bezug genommen wird auf den Verhaltenskodex der Interessenvertretung International Peace Operations Association (IPOA). Die derzeit 50 PSMFs umfassende IPOA (vgl. IPOA 2008) verfügt seit 2001 über einen Verhaltenskodex, der regelmäßig überarbeitet wird und aktuell (Stand: August 2008) in seiner elften Version vorliegt (vgl. IPOA 2006). ${ }^{7}$ Andere Formen der Selbstregulierung von PSMFs werden hier vernachlässigt. Dabei handelt es sich zum einen um die „Charter“ (BAPSC 2008a) der British Association of Private Security Companies (BAPSC), die jedoch eher als eine Vorstufe zu einem Verhaltenskodex zu sehen ist, welcher noch 2008 eingeführt werden soll (vgl. Schulz 2008: 160). Zum anderen haben sich einzelne PSMFs firmeninterne Verhaltenskodizes gegeben. Diese Kodizes orientieren sich jedoch vor allem an Fragen der Wirtschaftsethik

\footnotetext{
5 Für nähere Informationen s. http://www.eda.admin.ch/eda/de/home/topics/ intla/humlaw/pse/psechi.html (25.07.2008).

6 Für die Unterscheidung zwischen ,upscale firms “ und "downscale firms “ siehe Avant (2005: 221), vgl. auch Herbst (1999: 121ff.).
}

7 Diese Version trat am 1. Dezember 2006 in Kraft. 
und enthalten bis auf wenige Ausnahmen keine Regelungen für die Bereitstellung von Sicherheit im Einsatzgebiet, weshalb sie hier nicht berücksichtigt werden.

\section{2. "Upscale firms“ - „downscale firms"}

Als „upscale firms“ werden idealtypisch solche PSMFs bezeichnet, die nur für international anerkannte Kunden arbeiten, nur ausreichend qualifiziertes Personal entsenden, sich an internationale und nationale Rechtsnormen halten und diesen entsprechende Regeln zum Einsatz von Waffen etabliert haben. Die geringe Schwelle zum Eintritt in die Branche - schließlich können Personal und Gerät je nach Bedarf rekrutiert werden - sowie generell das hohe Maß an Intransparenz fördern jedoch die Entstehung und Existenz von sogenannten „downscale firms“ oder "fly-by-night companies“. Diese PSMFs bieten keine einsatzspezifischen Trainings an und stellen auch gering qualifiziertes Personal oder solches mit einer Vergangenheit in den Streitkräften von Diktaturen oder Apartheidsregimen ein.

Die Zuordnung einer Firma zum oberen („upscale firms“) oder zum unteren Segment („downscale firms“) der Branche ist auf Grund deren Intransparenz jedoch schwierig und oftmals erheblich von der Selbstdarstellung der Firmen beeinflusst. Firmen, die zum oberen Spektrum gehören oder hierzu gezählt werden möchten, sind sehr auf ein Image als legitimer Sicherheitsakteur bedacht. Ein solches Image ist wichtig für den Erhalt von Aufträgen, insbesondere von NGOs oder den UN. ${ }^{8}$ Legitimität wird hier in einem empirischen Sinne verstanden, d.h. Legitimität ist dann gegeben, wenn sie - in Abwesenheit von Zwang - von den jeweils Betroffenen anerkannt wird (vgl. Hall/Biersteker 2002: 4f.; Steffek 2003: 251ff.).

Um sich als legitime Sicherheitsakteure zu positionieren, verfolgen PSMFs verschiedene Strategien (vgl. Edwards/Zadek 2003: $211 \mathrm{ff}$.). Sie betonen erstens ihre Kompetenz. Hierzu verweisen sie auf die Qualifikation und Kompetenz ihres Personals. Entsprechend werben viele PSMFs auf ihren Websites mit der vorherigen militärischen Laufbahn ihrer Mitarbeiter, deren bei den regulären Truppen erreichten Positionen und erhaltenen Meriten. Zweitens betonen PSMFs immer wieder, dass sie eine Regulierung ihrer Arbeit befürworten, um den Kunden zu versichern, dass sie und vor allem ihre Mitarbeiter sich konform zu (inter-)nationalen Normen verhalten. So erklärt die Firma Aegis Defence Services auf ihrer Homepage: „Aegis and its Board, have long been supporters of Regulation of the PSC Sector" (Aegis 2008). Damit zusammenhängend betonen PSMFs drittens immer wieder die gemeinwohlorientierten Ziele ihrer Einsätze, namentlich Sicherheit, Stabilität und Frieden. Viertens spielen auch die Interessenvertretungen von PSMFs und deren Lobbyarbeit eine wichtige Rolle bei der Darstellung und Etablierung ihrer Mitgliedsfirmen als „upscale firms“. Neben den genannten IPOA und BAPSC gibt es Branchenverbände in Einsatzländern von PSMFs, bisher im Irak (PSCAI) und in Afghanistan (PSCAA). Insgesamt vertreten diese Verbände etwa ein Viertel der PSMFs. Aus dem Bestreben heraus, PSMFs als le-

8 Diese Akteure sind zwar nicht die finanzstärksten Kunden, aber die Arbeit für sie ist eine gute PR für PSMFs. gitime Sicherheitsakteure darzustellen, ist auch die Etablierung von Verhaltenskodizes zu verstehen.

\section{Verhaltenskodizes als Differenzierungs- merkmal}

Insofern sich die Etablierung eines Verhaltenskodexes positiv auf das Image einer Firma als legitimer Sicherheitsakteur auswirkt, kann Selbstregulierung als Differenzierungsmerkmal zwischen respektablen und weniger respektablen Firmen dienen (vgl. Percy 2006: 58; Schulz 2008: 161). Dies gilt umso mehr, als es keine staatliche Regulierung gibt, welche diese Aufgabe übernimmt. So heißt es auf der Homepage der BAPSC: „The Association believes that it is only through effective selfregulation that the Members will enhance their position and be able to achieve differentiation from non Members in the same industry sector" (BAPSC 2008a). Ziel ist es, Selbstregulierung als zentralen Wettbewerbsvorteil zu etablieren: „the ultimate incentive for self-regulation lies in the increase of competitiveness in the race of lucrative contracts with major clients" (Bearpark/Schulz 2007: 249). So heißt es in der Präambel des IPOA-Verhaltenskodexes:

„This Code of Conduct seeks to ensure the ethical standards of International Peace Operations Association member companies operating in conflict and post-conflict environments so that they may contribute their valuable services for the benefit of international peace and human security." (IPOA 2006)

Dabei können die an der Etablierung der Selbstregulierung beteiligten PSMFs Einfluss auf die Gruppe der konkurrenzfähigen Firmen nehmen. Je höher der Standard, desto weniger Firmen sind in der Lage, ihn zu erfüllen. Die Zahl dieser „upscale firms“ reduziert sich also mit der Höhe des Standards. Die Abgrenzung einzelner Unternehmen vom Rest der Branche, um für sich selbst Vorteile gegenüber Nicht-Mitgliedern bzw. weniger respektablen Firmen zu schaffen und sich somit den Erhalt von Aufträgen zu sichern, ist jedoch insofern problematisch, als dass dabei unter Umständen hohe Eintrittsbarrieren in den Markt festgesetzt werden Diese würden es neuen Unternehmen - je nach Höhe - schwer bis unmöglich machen, sich zu etablieren, weil sie ggf. nicht über die notwendigen Ressourcen verfügen, um beispielsweise ihren Mitarbeitern vorgeschriebene Trainingskurse anzubieten. Dabei besteht die Gefahr, dass ein hoher Standard die Nachfrage nicht befriedigen kann und Regierungen und IGOs doch wieder „downscale firms “ einkaufen, wodurch die trennende Funktion des Standards hinfällig werden würde. Bei der Etablierung des Standards muss also berücksichtigt werden, dass an der Nachfrage gemessen ausreichend Firmen in der Lage sein müssen, den Standard zu erfüllen, und zwar auch bei steigender Nachfrage. Zu diesem Zweck könnten zum Beispiel entsprechende Ressourcen (Trainingskurse etc.) von einem Verband zur Verfügung gestellt werden und die Kosten hierfür ggf. von allen Mitgliedern getragen werden.

Damit sich Selbstregulierung durch einen Verhaltenskodex positiv auf den Erhalt von Aufträgen auswirkt, müssen die Kunden Selbstregulierung als ein entscheidendes Kriterium bei der Vertragsvergabe anerkennen. Hierzu bedarf es zunächst einer 
höheren Transparenz der Branche, damit die Kunden zwischen einzelnen PSMFs differenzieren können. Bislang stellt der Markt den Kunden jedoch keine ausreichenden Informationen zur Verfügung, aufgrund derer die Qualität des Angebots einer Firma zeitnah beurteilt werden kann. So waren im Zuge des PSMF-Booms im Irak viele Kunden mit der Auswahl einer geeigneten PSMF schlichtweg überfordert. Die meisten Kunden hatten nicht nur noch nie mit einer solchen Firma zusammengearbeitet, sondern darüber hinaus überhaupt keinen Kontakt zur PSMF-Branche: „Often, the client's lack of experience meant that he would opt for the first provider he came across." (Donald 2006: 15) ${ }^{9}$

\section{Einflussnahme auf Auftragsvergabe durch die Kunden}

Haben Kunden die Möglichkeit, zwischen „upscale firms“ und „downscale firms“ zu unterscheiden und gibt es ausreichend „upscale firms“, um die Nachfrage nach deren Diensten zu erschwinglichen Preisen zu befriedigen, müssen schließlich die Kunden diese auch beauftragen wollen. Kunden können aber auch ein Interesse daran haben, gezielt „fly-by-night companies“ zu beauftragen. Jene werden in der Regel weniger wegen der Sozialverträglichkeit ihres Vorgehens, sondern wegen ihrer Effektivität und Effizienz und auch wegen ihrer Arbeit im Verborgenen angeworben (vgl. Bearpark/Schulz 2007: 247). Eine gewisse Anzahl von Kunden, die absichtlich dubiose Firmen unter Vertrag nimmt, wird es daher vermutlich immer geben. Entsprechend werden diese Firmen nicht verschwinden, auch nicht durch ein allgemeines Verbot von PSMFs. Ziel muss es aber sein, ihren Marktanteil so gering wie möglich zu halten.

Solange Selbstregulierung von den Kunden jedoch nicht als entscheidendes Kriterium für die Vertragsvergabe angesehen wird, weil diese dies nicht wollen oder die Intransparenz des Marktes dies verhindert, dient sie nur begrenzt der Einflussnahme von PSMFs auf die Marktstruktur und die Auftragsvergabe durch die Kunden. Dies verdeutlicht das Beispiel des IPOAVerhaltenskodexes. Für die Mitglieder der IPOA ist die US-Regierung einer der wichtigsten Kunden. Doch für die US-Regierung ist eine Mitgliedschaft in der IPOA bzw. eine Teilnahme an deren Verhaltenskodex kein diskriminierendes Kriterium für die Zusammenarbeit mit PSMFs. So arbeitet Blackwater auch nach dem Austritt aus der IPOA am 10. Oktober 2007 (vgl. IPOA 2007) weiterhin für das US-Außenministerium im Irak; die USRegierung hat den entsprechenden Vertrag mit Blackwater im April 2008 gar um ein weiteres Jahr verlängert (vgl. Hedgpeth 2008). Dies könnte sowohl mit der engen Verbindung der Firma zur US-Regierung als auch mit der strukturellen Abhängigkeit der US-Behörden von den Schutzdienstleistungen durch Blackwater zusammenhängen. Als Grund für den Austritt von Blackwater aus der IPOA wird eine drohende Untersuchung über Verstöße gegen den IPOA-Verhaltenskodex vermutet. Weitere Firmen, die kein IPOA-Mitglied sind und mit denen die US-Regierung Verträge unterhält oder unterhielt sind u.a.

9 Zu einer ähnlichen Schlussfolgerung kommt das South Eastern and Eastern Europe Clearinghouse for the Control of Small Arms and Light Weapons (SEESAC) für Postkonflikt-Situationen im Allgemeinen und Bosnien und Herzegowina im Speziellen (vgl. SEESAC 2006).
Aegis, Airscan, CACI, Global Strategies Group, Global Security Source, Kroll, ManTech International, Meteoric Tactical Solutions, Ronco, SOC-SMG und Titan (vgl. Isenberg 2004: 80ff.).

Somit ist die Einflussnahme der IPOA-Mitglieder auf die Begrenzung des Marktsegments der respektablen Firmen und die Auftragsvergabe durch einen ihrer Hauptkunden mittels ihres Verhaltenskodexes gering. Dies mag auch eine Erklärung dafür sein, dass der im IPOA-Verhaltenskodex enthaltene Standard so niedrig ist. Zwar enthält er für alle Mitgliedsfirmen verpflichtende Bestimmungen, u.a. zu Transparenz, zur Qualifikation der PSMF-Mitarbeiter, dem Gebrauch von Schusswaffen und der Zusammenarbeit mit anderen Akteuren im Einsatzgebiet. Insgesamt sind diese Bestimmungen jedoch vage und es fehlt an konkreten Handlungsanweisungen für die Mitgliedsfirmen. So sollen z.B. kleinere Vergehen der Angestellten von den Firmen selbst geregelt werden. Allerdings wird weder spezifiziert, was unter kleineren Vergehen zu verstehen ist, noch wie die Unternehmen damit umgehen sollen. Darüber hinaus sollen die IPOA-Mitgliedsfirmen im Falle eventueller Vertragsbrüche, Menschenrechtsverletzungen oder Verstößen gegen das Völkerrecht auch nur dann mit den jeweiligen Strafverfolgungsbehörden kooperieren, wenn es vertragliche und rechtliche Bestimmungen gestatten.

Vor dem Hintergrund der Nicht-Berücksichtigung des IPOAVerhaltenskodexes bei der Auftragsvergabe durch die US-Regierung ist es auch nicht wahrscheinlich, dass die IPOA bei der dieses Jahr anstehenden Überarbeitung ihres Verhaltenskodexes einen höheren Standard etabliert, selbst wenn ein signifikanter Teil der Mitgliedsfirmen ohne größere Probleme einen solchen erfüllen könnte. Noch unwahrscheinlicher ist, dass diese Firmen einen separaten Verhaltenskodex etablieren. Dies lohnt für diese Firmen nur dann, wenn die Kunden sich bei der Vertragsvergabe daran orientieren würden.

\section{Erhöhung der Einflussnahme durch Koregulie- rung und staatliche Sanktionierung}

Eine Berücksichtigung von Verhaltenskodizes als entscheidendes Kriterium bei der Auftragsvergabe könnte auf zweierlei Art und Weise sichergestellt werden. Eine Möglichkeit wäre die Beteiligung der Kunden an der Regulierung (Koregulierung). Denkbar wäre z.B. die gemeinsame Etablierung eines Verhaltenskodexes unter Beteiligung von Staaten, der Zivilgesellschaft und PSMFs. Ein entsprechender Standard müsste unabhängig von einzelnen Aufträgen sein, d.h. einen gewissen Grad an Allgemeingültigkeit erhalten und seine Einhaltung effektiv überwacht werden. Sind die Kunden von PSMFs an der Regelsetzung beteiligt, ist davon auszugehen, dass sie nur solche PSMFs unter Vertrag nehmen, welche sich der Einhaltung der gemeinsam etablierten Regeln verpflichtet haben. Eine andere Möglichkeit wäre die staatliche Sanktionierung der Selbstregulierung. Diese kann von einer öffentlichen Verlautbarung der Regierung bis hin zur Ex-post-Übernahme der Selbstregulierung in nationales Recht erfolgen. Jene könnte beispielsweise so beschaffen sein, dass Firmen - gleichgültig, ob sie ihre Dienstleistungen an inländische oder ausländische Kunden verkaufen - über einen bestimmten Verhaltenskodex verfügen oder Mitglied in einer 
Interessenvertretung sein müssen, die über einen solchen Kodex verfügt, um im Land ihres Firmensitzes von der dortigen Regierung eine Lizenz für den Verkauf ihrer Dienstleistungen erhalten zu können (vgl. Percy 2006: 60; Schulz 2008: 161).

Wenn Selbstregulierung von staatlicher Seite anerkannt würde, könnte dies eine Signalwirkung auch auf andere Kunden, v.a NGOs und IGOs, haben. Durch eine Übernahme privater Selbstregulierung in nationales Recht könnten andere Kunden auch dazu verpflichtet werden, nur PSMFs zu beauftragen, die sich einer Selbstregulierung unterziehen:

„Many client companies will never have hired security services before and have little idea of what to expect. It is only good sense to expect those clients to be reassured by the existence of an industry association, with a code of conduct, and by a legislative seal of approval." (Donald 2006: 33)

Dies bedeutet, dass eine staatliche Anerkennung der Selbstregulierung von PSMFs die Einflussnahme von den an der Etablierung der Selbstregulierung beteiligten PSMFs auf die Festlegung der ungefähren Anzahl der „upscale firms“, d.h. der konkurrenzfähigen PSMFs, und die Auftragsvergabe durch die Kunden vermutlich erhöhen würde. Wird private Selbstregulierung von PSMFs von staatlicher Seite anerkannt, könnte zudem auch die Höhe des in der Selbstregulierung enthaltenen Standards steigen. Diese Vermutung lässt sich leider nicht überprüfen, da kein solches Regulierungsinstrument existiert. Doch ist davon auszugehen, dass nicht nur der Nutzen für PSMFs steigen, sondern auch die Position des Regel setzenden Organs, zum Beispiel eines Verbandes, gestärkt würde, weil dieser nun den Firmen gegenüber höhere Anforderungen an Transparenz oder an die Qualifikation der Mitarbeiter besser rechtfertigen könnte. Hierbei handelt es sich wiederum um „key factors when trying to rid the private security industry of irresponsible clients “ (Bearpark/Schulz 2007: 244).

\section{Ausblick}

In den USA ist die staatliche Anerkennung einer Selbstregulierung von PSMFs unwahrscheinlich. Doch dies ist auch nicht das Ziel der Bemühungen der IPOA, erhalten deren Mitglieder doch den Großteil ihrer Verträge - gleichgültig, ob mit oder ohne Selbstregulierung - von der US-Regierung. Im Gegensatz dazu ist die BAPSC um eine staatlich anerkannte Selbstregulierung britischer PSMFs bemüht: „the BAPSC and its members recognise that their objectives will best be achieved through effective self regulation in partnership with the UK Government and International Organisations" (BAPSC 2008b). Den Mitgliedern der BAPSC würde die staatliche Anerkennung eines Verhaltenskodexes die Aufträge der Regierung und vermutlich auch anderer Kunden (Unternehmen, NGOs) sichern (vgl. Bearpark/Schulz 2007; Donald 2006: 32). Bislang ist in Großbritannien aber kein politischer Wille zur staatlichen Anerkennung einer Selbstregulierung durch die BAPSC erkennbar. Es mangelt zudem an ausreichendem Druck von Seiten zivilgesellschaftlicher Akteure, um die Regierung zu einem solchen Schritt zu bewegen. Insgesamt darf die Diskussion über Selbstregulierung aber nicht diejenige über nationale Gesetze ver- drängen. Letztere sind nach wie vor das wichtigste Instrument zur Regulierung von PSMFs.

\section{Literatur:}

Aegis (2008): Frequently Asked Questions. URL: http://www. aegisworld.com/aegis-faq.html; 18.05.2008.

Avant, Deborah (2005): The Market for Force. The Consequences of Privatizing Security. Cambridge et al.

BAPSC (2008a): Charter. URL: http://www.bapsc.org.uk/key_ documents-charter.asp, 10.04.2008.

BAPSC (2008b): Home. URL: http://www.bapsc.org.uk/, 10.04.2008.

Bearpark, Andrew und Sabrina Schulz (2007): The future of the market. In: Chesterman, Simon und Chia Lehnardt (Hrsg.): From Mercenaries to Market. The Rise and Regulation of Private Military Companies. Oxford, New York. S. 239-250.

Bjork, Kjell und Richard Jones (2005): Overcoming Dilemmas Created by the 21st Century Mercenaries: conceptualising the use of private security companies in Iraq. In: Third World Quarterly. 26: 4-5, S. 777-796.

Broder, John M. und James Risen (2007): Armed Guards in Iraq Occupy a Legal Limbo. In: The New York Times. 20. September 2007. URL: http://www.nytimes.com/2007/09/20/ world $/$ middleeast $/ 20$ blackwater.html? pagewanted=print, 10.04.2008.

Caparini, Marina (2007): Domestic Regulation. Licensing regimes for the export of military goods and services. In: Chesterman, Simon und Chia Lehnardt (Hrsg.): From Mercenaries to Market. The Rise and Regulation of Private Military Companies. Oxford et al., S. 158-178.

Donald, Dominick (2006): After the Bubble. British Private Security Companies After Iraq. London. Whitehall Paper Nr. 66.

Edwards, Michael und Simon Zadek (2003): Governing the Provision of Global Public Goods: The Role and Legitimacy of Nonstate Actors. In: Kaul, Inge et al. (Hrsg.): Providing Global Public Goods. Managing Globalization. New York, Oxford. S. 200-224.

Finer, Jonathan (2005): Security Contractors in Iraq Under Scrutiny After Shootings. In: Washington Post, 10. September 2005. URL: http://www.washingtonpost.com/wp-dyn/content/article/2005/09/09/AR2005090902136_pf.html, 10.04.2008

Hall, Rodney Bruce und Thomas J. Biersteker (2002): The emergence of private authority in the international system. In: Hall, Rodney Bruce und Thomas J. Biersteker (Hrsg.): The Emergence of Private Authority in Global Governance. Cambridge. S. 321.

Hedgpeth, Dana (2008): State Department to Renew Deal With Blackwater for Iraq Security. In: Washington Post, 05. April 2008. URL: http://www.washingtonpost. com/wp-dyn/content/article/2008/04/04/AR200804 0403449_pf.html, 11.04.2008. 
Herbst, Jeffrey (1999): The Regulation of Private Security Forces. In: Mills, Greg and John Stremlau (Hrsg.): The Privatisation of Security in Africa. Johannesburg. S. 107-127.

Holmqvist, Caroline (2005): Private Security Companies. The Case for Regulation. SIPRI Policy Paper No. 9, URL: http://editors.sipri.se/pubs/SIPRI_PolicyPaper9.pdf, 10.04.2008

IPOA (2006): Code of Conduct. URL: http://ipoaworld.org/eng/ codeofconductv11eng.html, 08.08.2008

IPOA (2007): IPOA Press Release. Statement Regarding the Membership Status of Blackwater USA. 12. Oktober 2007. URL: http://ipoaonline.org/php/index.php?option=com_content\& task=view\&id=156\&Itemid=80, 15.05.2008.

IPOA (2008): Current IPOA Corporate Membership List. URL: http://ipoaworld.org/eng/currentmembers.html, 08.08.2008.

Isenberg, David (2004): A Fistful of Contractors: The Case for a Pragmatic Assessment of Private Military Companies in Iraq. URL: http://www.basicint.org/pubs/Research/2004PMC.pdf, 18.05.2008 (Research Report Nr. 4/2004. British American Security Information Council).

Johnston, David und John M. Broder (2007): F.B.I. Says Guards Killed 14 Iraqis Without Cause. In: The New York Times. 14. November 2007. URL: http://www.nytimes.com/2007/11/14/ world/middleeast $/ 14$ blackwater.html?ei=5088\&en=baf513ff1 e78a9fd\&ex=1352696400\&partner=rssnyt\&emc=rss\&pagewa nted=print, 10.04.2008.

Messner, J. J. und Ylana Gracielli (2007): State of the Peace and Stability Operations Industry. Second Annual Survey 2007. URL: http://peaceops.org/poi/images/stories/poi_rp_industrysurvey2007.pdf, 10.04.2008.

Miller, Christian T. (2005): Private Security GuardsOperate with Little Supervision. In: Los Angeles Times. 4. Dezember
2005. URL: http://www.globalpolicy.org/security/issues/iraq/ reconstruct/2005/1204supervision.htm, 10.04.2008.

Percy, Sarah (2006): Regulating the Private Security Industry. Adelphi Paper Nr. 384.

Schreier, Fred und Marina Caparini (2005): Privatising Security: Law, Practice and Governance of Private Military and Security Companies. Geneva Centre for the Democratic Control of Armed Forces (DCAF), Occasional Paper - No 6. URL: http://www. dcaf.ch/_docs/op06_privatising-security.pdf; 18.05.2008.

Schulz, Sabrina (2008): Private Sicherheit als Exportschlager? Die private Sicherheitsindustrie in Großbritannien im Spannungsfeld freier Marktkräfte und internationaler Rechtsgüter. In: Feichtinger, Walter et al. (Hrsg.): Private Sicherheits- und Militärfirmen. Konkurrenten - Partner - Totengräber? Wien et al. S. 147-161.

SEESAC (2006): The Sarajevo Client Guidelines for the Procurement of Private Security Companies. URL: http://www.seesac. org/reports/Procurement\%20guidelines.pdf, 09.04.2007.

Singer, Peter Warren (2004): War, Profits, and the Vacuum of Law: Privatized Military Firms and International Law. In: Columbia Journal of Transnational Law 42: 2. S. 521-549.

Singer, Peter Warren (2006): Die Kriegs-AGs. Frankfurt am Main.

Singer, Peter Warren (2007): Can't Win With 'Em, Can't Go To War Without 'Em: Private Military Contractors and Counterinsurgency. Foreign Policy at Brookings Policy Paper Number 4, September 2007. URL: http://www3.brookings.edu/fp/research/singer200709.pdf, 09.10.2007.

Steffek, Jens (2003): The Legitimation of International Governance: A Discourse Approach. In: European Journal of International Relations. 9: 2. S. 249-275.

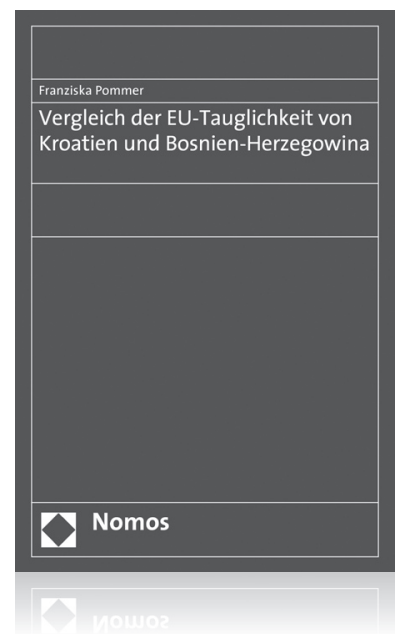

\section{Vergleich der EU-Tauglichkeit von Kroatien und Bosnien-Herzegowina Von Franziska Pommer \\ 2008, 226 S., brosch., 44,-€, ISBN 978-3-8329-3644-O}

Der südosteuropäische Raum ist eine der größten Herausforderungen und Chancen für die europäische Staatengemeinschaft. Die Studie zeigt, wo derzeit die Probleme in Kroatien und Bosnien-Herzegowina liegen und welche Lösungen zum Erfolg führen können. Zudem werden die Anforderungen, die die EU an ihre zukünftigen Mitglieder stellt, analysiert und hinterfragt. 